\title{
Low and high velocity clouds produced by young stellar clusters
}

\author{
A. Rodríguez-González ${ }^{1}$, A. C. Raga ${ }^{1}$, and J. Cantó ${ }^{2}$ \\ 1 Instituto de Ciencias Nucleares, Universidad Nacional Autónoma de México, Ap. 70-543, México, D.F., CP: 04510, México \\ e-mail: [ary;raga]@nucleares.unam.mx \\ 2 Instituto de Astronomía, Universidad Nacional Autónoma de México, Ap. 70-468, México, D.F., CP: 04510, México
}

Received 20 January 2009 / Accepted 5 April 2009

\section{ABSTRACT}

\begin{abstract}
Context. Intermediate and high velocity HI clouds rain onto the plane of our Galaxy. They are observed at heights of between 500 and $1500 \mathrm{pc}$, falling onto the Galactic plane at velocities from 50 to $140 \mathrm{~km} \mathrm{~s}^{-1}$.

Aims. To explain the origin of these clouds, we present a galactic fountain model, driven by the wind from a super stellar cluster (SSC).

Methods. We solve the equations for a steady, radiative de Laval nozzle flow. We consider two effects not considered previously in astrophysical nozzle flow models: cooling functions for different metallicities, and the direct action of the galactic gravitational field on the gas flowing along the nozzle.

Results. For an adiabatic nozzle flow, the gravity acting directly on the gas within the nozzle "stalls" the nozzle flow for initial wind velocities lower than the escape velocity from the Galaxy. For the same wind velocity, a radiative nozzle flow stalls at lower altitudes above the galactic plane. We find that SSC winds with velocities of $v_{\mathrm{w}}=500-800 \mathrm{~km} \mathrm{~s}^{-1}$ produce nozzles stall at heights of $x_{\mathrm{m}}=1-15 \mathrm{kpc}$. The stalled nozzle flow then rains back onto the galactic plane at velocities in the range observed in intermediate and high velocity HI clouds.

Conclusions. We study a nozzle flow driven by a wind from a SSC close to the Galactic centre. We find that for velocities within the range expected for a SSC wind, we can produce nozzle flows that stall above the galactic plane. These stalled flows produce cool, infalling clouds with velocities similar to those of intermediate and high velocity HI clouds.
\end{abstract}

Key words. hydrodynamics - ISM: jets and outflows - galaxies: star clusters - galaxies: starburst - galaxies: intergalactic medium

\section{Introduction}

The observations of Munch \& Zirin (1691) showed absorption (mostly Ca II) lines of interstellar clouds at distances of 500$1500 \mathrm{pc}$ above the Galactic plane. These high altitude interstellar clouds have been described by many authors as neutral hydrogen (HI) clouds at distances of between $300 \mathrm{pc}$ and $12 \mathrm{kpc}$ with infall velocities between -50 and $-140 \mathrm{~km} \mathrm{~s}^{-1}$.

The clouds have been divided into two categories: intermediate-velocity clouds (IVCs) and high-velocity clouds (HVCs). IVCs have radial velocities in the range of $30 \mathrm{~km} \mathrm{~s}^{-1}$ $\leq\left|v_{\mathrm{LSR}}\right| \leq 90 \mathrm{~km} \mathrm{~s}^{-1}$ with respect to the LSR, and HVCs have radial velocities $\left|v_{\text {LSR }}\right| \geq 90 \mathrm{~km} \mathrm{~s}^{-1}$. In the Galaxy (and in other galaxies), the IVCs have typical altitudes of between $300 \mathrm{pc}$ and $2.5 \mathrm{kpc}$, and masses of up to $\sim 10^{4} M_{\odot}$. HVCs have been observed at higher altitudes (of up to $\sim 12 \mathrm{kpc}$ ). Several complexes of HVCs with masses of $\sim 10^{5}-10^{6} M_{\odot}$ have been observed (see, e. g., Wakker \& van Woerden 1997). The general characteristics of HI clouds in the Galaxy were discussed by Dickey \& Lockman (1990) and Wakker \& van Woerden (1997). Their general chemical properties were described by Lu et al. (1998), Wakker et al. (1999), Murphy et al. (2000), Sembach et al. (2000), and Bluhm et al. (2001).

Significant progress has been made in exploring the distribution of IVCs and HVCs in the halo of the Galaxy (see, e.g., Dettmar 2005). However, the origin of these clouds is unclear. Oort (1970) proposed that high altitude clouds (in the Galaxy) are formed in past episodes of star formation. Several authors have used this scenario to explain the origin of the IVCs.
However, the HVCs have been considered to be extragalactic objects that are about to merge with the Galaxy (Fraternali \& Binney 2006, Melioli et al. 2008). An alternative scenario was proposed by Blitz et al. (1999), who suggested that HVC and IVC could be relics of the early stages of Galactic formation.

It has also been proposed that both IVCs and HVCs are formed in the disk of our galaxy, in the so-called "galactic fountains". These models were described by Shapiro \& Field (1976), Houck \& Bregman (1990), and de Gouveia Dal Pino et al. (2009). In this scenario, the cloud formation is fed by chimneys of hot, ascending gas that cools and falls back onto the Galactic disk in the form of discrete clouds. Observations indicate that chimneys are indeed generated in other galaxies by multiple supernovae ( $\mathrm{SNe}$ ) explosions (Veilleux et al. 2005; Konstantopoulos et al. 2008).

De Gouveia Dal Pino et al. (2009) showed that SNe explosions can lead to the formation of hot superbubbles that drive a supersonic wind out of the galactic disk. From 3D, radiative hydrodynamical simulations, they found that the galactic fountains driven by SNe may reach altitudes of up to $\sim 5 \mathrm{kpc}$ in the halo and thus allow the formation of IVCs. However, the HVCs (found at higher altitudes) require a different production mechanism.

We propose that HVCs (and possibly also some of the IVCs) could be formed by fountains driven by super stellar clusters (SSCs). SSCs are dense clusters of young massive stars. These stellar clusters contain hundreds or thousands of very young, energetic stars, and have stellar densities far greater than those seen in normal OB associations. These star clusters have ages of $\sim 1-10 \mathrm{Myr}$, radii of $\sim 1-10 \mathrm{pc}$, total cluster masses 
of $10^{3}-10^{6} M_{\odot}$ (Melo et al. 2005, reported a mean mass per stellar cluster of $\sim 2 \times 10^{5} M_{\odot}$, for M 82), and typical cluster wind velocities of 500-1500 $\mathrm{km} \mathrm{s}^{-1}$ range (see also, Silich et al. 2004). The central stellar densities of SSCs have values of up to $\sim 10^{5} M_{\odot} \mathrm{pc}^{-3}$. However, SSCs with older ages and/or higher masses do exist (Walcher et al. 2006, reports a cluster with $\left.6 \times 10^{7} M_{\odot}\right)$. On the other hand, the metallicity of the gas reinserted (via supernova explosions and stellar winds) into the stellar cluster medium, reaches supersolar values $(1 \leq Z \leq 14$, see also Meynet \& Maeder 2002; and Tenorio-Tagle et al. 2003) during most of the cluster evolution.

SSCs have been observed in a wide range of star-forming galaxies, such as merging systems (NGC 4038/4039, Whitmore \& Schweizer 1995), dwarf galaxies (Henize 2-10, Johnson et al. 2000), classical starbursts (M 82, Gallagher \& Smith 1999; Melo et al. 2005), as well as in the Galaxy (Arches Cluster: Nagata et al. 1995; Cotera et al. 1996; Serabyn et al. 1998) amongst many other systems (for a review, see Whitmore 2000).

In this paper, we present a model for the formation of low and high velocity HI clouds by means of radiative nozzle flows driven by the wind from a SSC. The nozzle flow is produced by the interaction of the shocked SSC wind with the stratified halo and the gravitational potential of the Galaxy. In our model, we assume that the SSC that produces to the fountain is close to the Galactic centre. This is an appropriate assumption if we consider the wind from the Arches cluster or from other possible massive (but still undetected) stellar clusters close to the Galactic centre. In galaxies such as M 82 and NGC 253, it appears that we do see outflows ejected from SSC's in the central regions of these galaxies (see Rodríguez-González et al. 2008).

The paper is organized as follows. In Sect. 2, we describe the equations for a radiative nozzle flow, and in Sect. 3 we discuss the analytic solution obtained in the adiabatic case. In Sect. 4, we describe the Galactic potential and hot gas distribution, which are used in Sect. 5 to compute numerical integrations of the radiative nozzle flow equations. Finally, in Sect. 6 we discuss the application to these models for low and high velocity HI cloud formation, and we present our conclusions in Sect. 7.

\section{Equations for a radiative nozzle flow}

We assume that the interaction of a wind from a SSC (located close to the centre of the galaxy) with the surrounding interstellar medium (ISM) leads to the formation of an approximately spherical shock. The thermalized, post-shock material is then channeled into two oppositely directed nozzles because of the pressure stratification of the environmental material. In the context of the generation of jets by active galactic nuclei, this scenario was first explored by Blandford \& Rees (1974)

We consider a one-dimensional, stationary, radiative nozzle flow under the action of a gravitational potential. We first write the energy equation in the "entropy conservation" form

$$
\frac{\mathrm{d}}{\mathrm{d} x} P \rho^{-\gamma}=-\frac{(\gamma-1) L}{\rho^{\gamma} u} .
$$

where $x$ is the direction of propagation of the flow, $P$ is the gas pressure, $\rho$ the density, $u$ the flow velocity, $\gamma$ the specific heat ratio, and $L$ is the energy loss per unit time and volume. We now consider the momentum equation in the form

$$
\frac{1}{\rho} \frac{\mathrm{d} P}{\mathrm{~d} x}=-\frac{\mathrm{d}}{\mathrm{d} x}\left[\frac{u^{2}}{2}+\Phi(x)\right],
$$

where $\Phi(x)$ is the gravitational potential, which we take to be a known function. Equations (1) and (2) can be combined to obtain

$\frac{\mathrm{d}}{\mathrm{d} x}\left[\frac{u^{2}}{2}+\frac{c_{\mathrm{s}}^{2}}{\gamma-1}+\Phi(x)\right]=-\frac{L}{\rho u}$,

with

$c_{\mathrm{s}}^{2}=\frac{\gamma P}{\rho}$.

Equation (3) simplifies to Bernoulli's theorem for the case of a non-radiative flow (i.e., for $L=0$ ).

Following Blandford \& Rees (1974), we now assume that the nozzle flow has a slowly varying cross-section, so that the pressure of the flow always adjusts to a value close to the pressure $P_{\mathrm{e}}(x)$ of the surrounding environment. If one also knows the cooling function $L$ as a function of the density and temperature of the flow, the nozzle flow can then be obtained by integrating the system described by Eqs. (1) and (3).

The initial conditions for this integration are obtained by assuming that the initially isotropic wind from the cluster (of massloss rate $\dot{M}$ and terminal velocity $v_{\mathrm{w}}$ ) follows a strong, spherical shock before flowing along the nozzle. Assuming that the shock is strong, we have a post-shock velocity

$v_{\mathrm{ps}}=\frac{\gamma-1}{\gamma+1} v_{\mathrm{w}}$

and a post-shock sound speed

$c_{\mathrm{ps}}=\frac{\sqrt{2 \gamma(\gamma-1)}}{\gamma+1} v_{\mathrm{w}}$.

Following Raga \& Cantó (1989), we assume that the initial velocity and sound speed along the nozzle flow are $v_{0} \approx v_{\mathrm{ps}}$ and $c_{0} \approx c_{\mathrm{ps}}$, respectively (see Eqs. (5) and (6)). The initial density $\rho_{0}=\rho(x=0)$ of the nozzle flow can be obtained from the $P(x)=P_{\mathrm{e}}(x)$ lateral pressure-balance condition (see above)

$\rho_{0}=\frac{\gamma P_{\mathrm{e}}(0)}{c_{\mathrm{ps}}^{2}}$,

where $P_{\mathrm{e}}(0)$ is the (known) environmental pressure at $x=0$, and $c_{\mathrm{ps}}$ is given by Eq. (6). The initial conditions for the integration of the nozzle flow problem (Eqs. (1) and (3)) are then given by Eqs. (5)-(7).

Finally, the mass conservation equation for a flow of slowly varying cross-section

$\dot{M}=\pi R^{2} \rho u$,

where $\dot{M}$ is the mass-loss rate, can be used to calculate the cylindrical radius $R$ of the cross-section as a function of position $x$ along the nozzle flow.

\section{The adiabatic solution}

For a non-radiative flow (i.e., with $L=0$ ), Eq. (3) can be trivially integrated to obtain

$\frac{u^{2}}{2}+\frac{c_{\mathrm{s}}^{2}}{\gamma-1}+\Phi(x)=\frac{v_{\mathrm{w}}^{2}}{2}+\Phi_{0}$,

where $\Phi_{0}=\Phi(x=0)$, and the constant on the right is obtained by setting $u=v_{\mathrm{ps}}$ and $c_{\mathrm{s}}=c_{\mathrm{ps}}$ (see Eqs. (5) and (6)) at $x=0$. 
For $L=0$, from Eqs. (9), (2), (4) and (6) and the lateral pressure balance condition, we obtain the solution:

$u^{2}(x)=v_{\mathrm{w}}^{2}\left\{1-\frac{4 \gamma}{(\gamma+1)^{2}}\left[\frac{P_{\mathrm{e}}(x)}{P_{0}}\right]^{(\gamma-1) / \gamma}\right\}+2\left[\Phi_{0}-\Phi(x)\right]$,

where $P_{\mathrm{e}}(x)$ is the environmental pressure stratification, and $\Phi(x)$ is the gravitational potential. If these two functions are known, Eq. (10) directly gives the velocity along the nozzle flow as a function of position $x$. We should note that this solution is equivalent to the one of Blandford \& Rees (1974), but also includes the direct effect of the gravitational force on the material within the nozzle flow.

It is easy to check that for $x=0$, Eq. (10) correctly gives $u(0)=c_{\mathrm{ps}}$ (see Eq. (6)), the imposed initial value for the flow velocity. For $x \rightarrow \infty$, for all finite mass environmental stratifications we have $P_{\mathrm{e}}(x) \rightarrow 0$ and $\Phi(x) \rightarrow 0$ (setting the zero of the potential at infinity). We then obtain

$u(x) \rightarrow u_{\infty}=\sqrt{v_{\mathrm{w}}^{2}+2 \Phi_{0}}$, for $x \rightarrow \infty$.

Because $\Phi_{0}<0$, this equation clearly has a physical solution only if

$v_{\mathrm{w}}>v_{\mathrm{esc}}=\sqrt{-2 \Phi_{0}}$,

in other words, the nozzle flow extends out to infinity only if the velocity of the wind (which is producing the nozzle flow) exceeds the escape velocity. If this condition is not met, the nozzle flow reaches a maximum height $x_{\mathrm{m}}$ (which can be computed from Eq. (10) setting $u=0$ ), at which it stalls. However, since the escape velocity from the centre of our galaxy has a value $v_{\mathrm{esc}} \approx 700 \mathrm{~km} \mathrm{~s}^{-1}$, a wind from a cluster of massive stars (with $v_{\mathrm{w}} \sim 1000 \mathrm{~km} \mathrm{~s}^{-1}$ ) satisfies the condition shown in Eq. (12), and therefore produces an adiabatic nozzle flow that escapes from the Galaxy. In the following sections, we show that this is not necessarily the case for radiative nozzle flows.

\section{The Galactic potential and hot gas distribution}

We assume that the halo has a uniform temperature. An isothermal halo is an assumption that is consistent with the observed diffuse, soft X-ray background emision (Garmire et al. 1992). The Galactic gas density $\rho_{\mathrm{g}}$ and thermal pressure $P_{\mathrm{g}}$ are given by (Espresate et al. 2002)

$\rho_{\mathrm{g}}(x)=\rho_{\mathrm{g}, 0} \exp ^{\left[\Phi_{0}-\Phi(x)\right] / c_{\mathrm{g}}^{2}}$,

and,

$P_{\mathrm{g}}(x)=P_{0} \exp ^{\left[\Phi_{0}-\Phi(x)\right] / c_{\mathrm{g}}^{2}}$,

where, $P_{0}=\rho_{\mathrm{g}, 0} c_{\mathrm{g}}^{2}$ and $\rho_{\mathrm{g}, 0}$ are the pressure and density, (respectively), of the hot gas at the Galactic centre, and $c_{\mathrm{g}}$ is the (uniform) isothermal sound speed of the halo. For the gravitational potential $\Phi(x)$, we use the model proposed by Allen \& Santillán (1991) and Espresate et al. (2002). These authors defined three components for the Galactic mass:

(1) a nucleus, represented by a spherically symmetric distribution;

(2) a disk component;

(3) a spherical, massive halo. Hence; the gravitational potential is given by the sum of these three contributions.

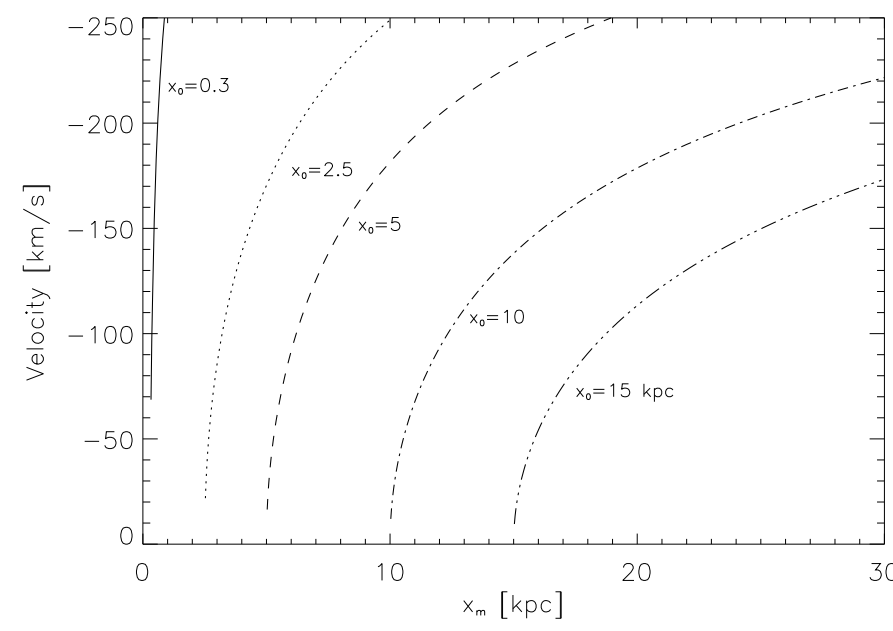

Fig. 1. Free-fall velocity as a function of $x_{\mathrm{m}}$ for different final heights $x_{0}=0.3,2.5,5,10$ and $15 \mathrm{kpc}$ (solid, dotted, dashed, dash-dot, and dash-dot-dot lines, respectively).

The units are such that if $x$ is in kiloparsecs and $M_{1}, M_{2}$, and $M_{3}$ are in Galactic mass units $\left(\mathrm{gmu}=2.32 \times 10^{7} M_{\odot}\right)$, the units for $\Phi(x)$ are $100 \mathrm{~km}^{2} \mathrm{~s}^{-2}$ (Espresate 2002). The equations for the potentials along the rotation axis of the Galaxy, for the different components are as follows

1. the nucleus is represented by

$\Phi_{1}(x)=\frac{M_{1}}{\left(x^{2}+b_{1}^{2}\right)^{1 / 2}}$,

where, $M_{1}=606 \mathrm{gmu}$ and $b_{1}=0.3873 \mathrm{kpc}$;

2. the disk component is represented by

$\Phi_{2}(x)=\frac{M_{2}}{a_{2}+\left(x^{2}+b_{2}^{2}\right)^{1 / 2}}$,

where, $M_{2}=3690.0 \mathrm{gmu}, a_{2}=5.3178 \mathrm{kpc}$, and $b_{2}=0.25 \mathrm{kpc} ;$ and,

3. finally, a halo truncated at $100 \mathrm{kpc}$ (see also Allen \& Santillán 1991), has a potential

$\Phi_{3}(x)=b_{3}\left(1+\ln \left[1+\left(\frac{x}{a_{3}}\right)^{1.02}\right]\right)-c_{3}$,

where, $a_{3}=12.0 \mathrm{kpc}, b_{3}=377.04 \mathrm{gmu} / \mathrm{kpc}$, and $c_{3}=$ $1201 \mathrm{gmu} / \mathrm{kpc}$. The total gravitational potential at any point $x$ along the rotation axis of the galaxy is then given by

$\Phi(x)=\Phi_{1}(x)+\Phi_{2}(x)+\Phi_{3}(x)$.

This expression for the gravitational potential (from Espresate et al. 2002) implies a total mass for the Galaxy of $M_{\mathrm{T}}=$ $9.0 \times 10^{11} M_{\odot}$, and a local circular velocity of $220 \mathrm{~km} \mathrm{~s}^{-1}$ and $r_{\odot}=8.5 \mathrm{kpc}$. With this potential, we can compute the free-fall velocity of material injected at a height $x_{\mathrm{m}}$ above the galactic plane (by a nozzle flow originating close to the galactic centre). By the time that the material injected (with a velocity $\approx 0$ ) at $x_{\mathrm{m}}$ has fallen to a height $x_{0}$ above the galactic plane, it has acquired a velocity:

$v_{\mathrm{f}}^{2}=\Phi\left(x_{\mathrm{m}}\right)-\Phi\left(x_{0}\right)$.

Figure 1 shows $v_{\mathrm{f}}$ as a function of $x_{\mathrm{m}}$ for different final heights $x_{0}$, for values of $x_{0}$ that are typical of the observed altitudes of IVCs and HVCs. The resulting values of $v_{\mathrm{f}}$ are used 


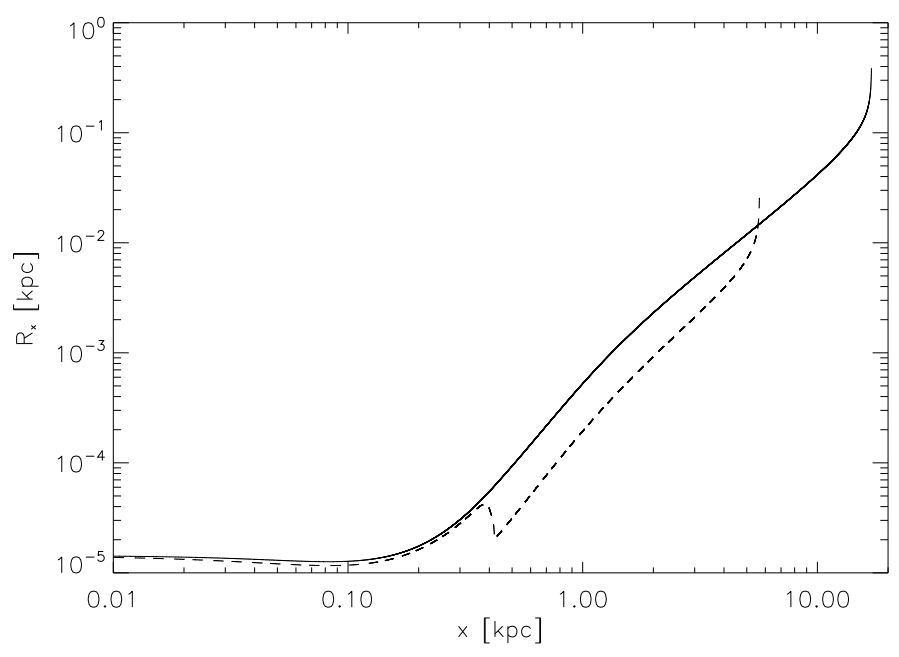

Fig. 2. The radius of the nozzle flow cross-section as a function of height $x$ above the galactic plane. The solid line shows the $Z=1$ model and the dashed line shows $Z=5 Z_{\odot}$ model.

in the following sections to determine the conditions that have to be satisfied by nozzle flows to produce clouds with velocities similar to those of IVCs and HVCs.

\section{The models}

We consider a nozzle flow fed by a wind from a massive stellar cluster situated close to the Galactic centre. The wind travels through a shock and then forms a nozzle aligned with the rotation axis of the Galaxy, which will is to the gravitational potential given by Eq. (18), and the environmental pressure stratification given by Eqs. (13), (14).

We fix the environmental pressure stratification and the gravitational potential as described in the previous section by assuming a temperature of $10^{6} \mathrm{~K}$ and a density on the galactic plane of $0.5 \mathrm{~cm}^{-3}$ for the hot halo. The remaining free parameters of the model are then the cluster wind velocity $v_{\mathrm{w}}$, its mass-loss rate $\dot{M}$, and metallicity $Z$. The initial flow velocity, sound speed, and density of the flow are functions of only the wind velocity $v_{\mathrm{w}}$ and the pressure of the hot environmental gas close to the galactic plane (see Eqs. (5)-(7)). The metallicity $Z$ is important for determining the cooling rate. The mass-loss rate is only important for determining the radius of the nozzle flow crosssection (see Eq. (8)), so that we choose a single mass-loss rate $\dot{M}=10^{-2} M_{\odot} \mathrm{yr}^{-1}$ for all models.

This is a reasonable mass-loss rate in the following sense. To reach a height of $\sim 10 \mathrm{kpc}$ above the galactic plane, the nozzle flow must exist for a time $\sim 10 \mathrm{kpc} / v_{\mathrm{w}} \sim 10 \mathrm{Myr}$ (where $v_{\mathrm{W}} \sim 1000 \mathrm{~km} \mathrm{~s}^{-1}$ approximate cluster wind velocity). This timescale coincides with the lifetime of the stellar population of a SSC (Leitherer \& Heckman 1995). During this lifetime, a cluster of $\sim 10000$ massive stars (with a total mass of $\sim 10^{6} \mathrm{M}_{\odot}$ ) will have lost $\sim 10 \%$ of its initial mass. If we consider a much smaller cluster such as, e.g., the Arches cluster near the Galactic centre (which has only 100 massive stars), it would be necessary to consider a much lower mass-loss rate $\left(\sim 10^{-4} M_{\odot} \mathrm{yr}^{-1}\right)$. However, the mass-loss rate from the cluster enters only in the calculation of the cylindrical radius of the nozzle flow (see Eq. (8) and Fig. 2), and the dynamical characteristics of the flow do not depend on the value of the mass loss.

We should note that a cluster of $10^{4}-10^{5}$ massive stars will feed a $\sim 10^{5}-10^{6} M_{\odot}$ nozzle flow, which in principle could

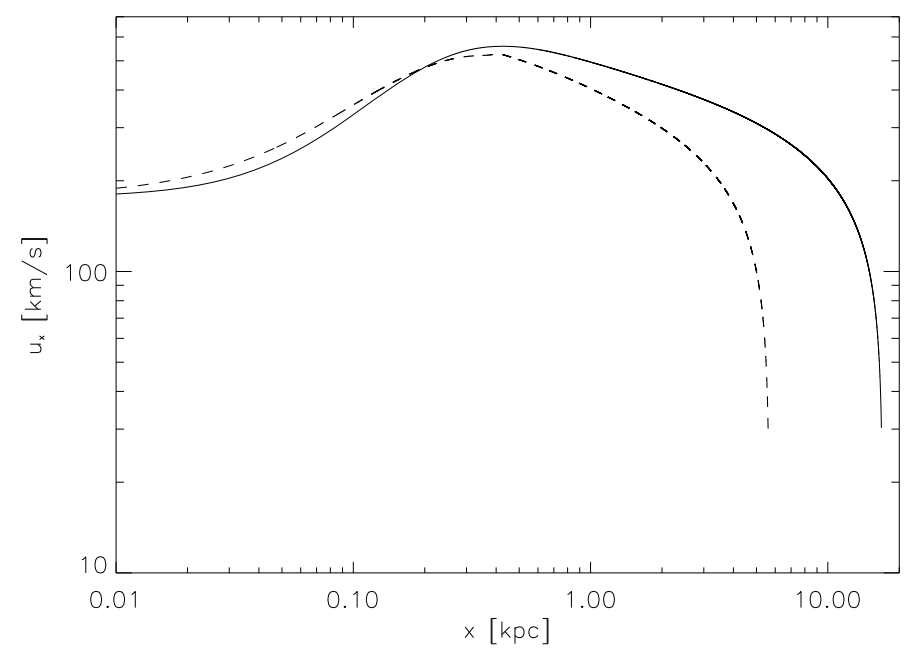

Fig. 3. The gas velocity as a function of height $x$ above the galactic plane. The lines have the same description as Fig. 2.

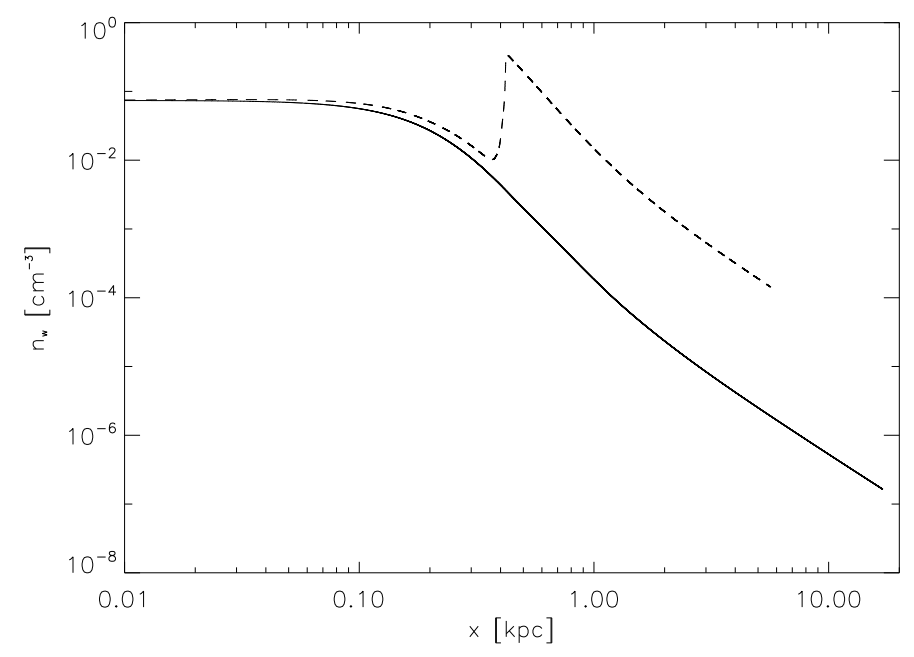

Fig. 4. The numerical gas density as a function of height $x$ above the galactic plane. The lines have the same description of Fig. 2.

produce the mass observed in an HVC complex (see Wakker \& van Woerden 1997). A cluster with 100-1000 massive stars will produce a $\sim 10^{3}-10^{4} M_{\odot}$ nozzle flow, corresponding to the typical mass of an IVC (see de Gouveia Dal Pino et al. 2009).

We now carry out numerical integrations of Eqs. (1) and (3) with the initial conditions given by Eqs. (5)-(7) for different values of $v_{\mathrm{w}}$ and $Z$. We use the cooling function of Raymond et al. (1976), who tabulated the cooling rate as a function of the temperature $T$ and metallicity $Z$ of the gas (a density dependence $\propto \rho^{2}$, appropriate to a low-density regime cooling, is assumed). Figures $2-5$ show the results obtained for models with $v_{\mathrm{w}}=700 \mathrm{~km} \mathrm{~s}^{-1}$ and $Z=1$, and 5 solar metallicities. Figure 2 shows the radius of the nozzle flow cross-section as a function of height $x$ above the galactic plane. The $Z=1$ model (solid line) shows a radius that decreases for $x<0.10 \mathrm{kpc}$, and then increases monotonically at greater heights. This behaviour is similar to that found in adiabatic nozzles, in which the nozzle radius decreases in the region of subsonic flow, and increases with distance after the sonic transition. The radius of the nozzle flow diverges at $x_{\mathrm{m}} \approx 15 \mathrm{kpc}$. This divergence was not found in previous nozzle solutions (see, e.g., Blandford \& Rees 1974; Raga \& Cantó 1989), because they did not include the effect of 


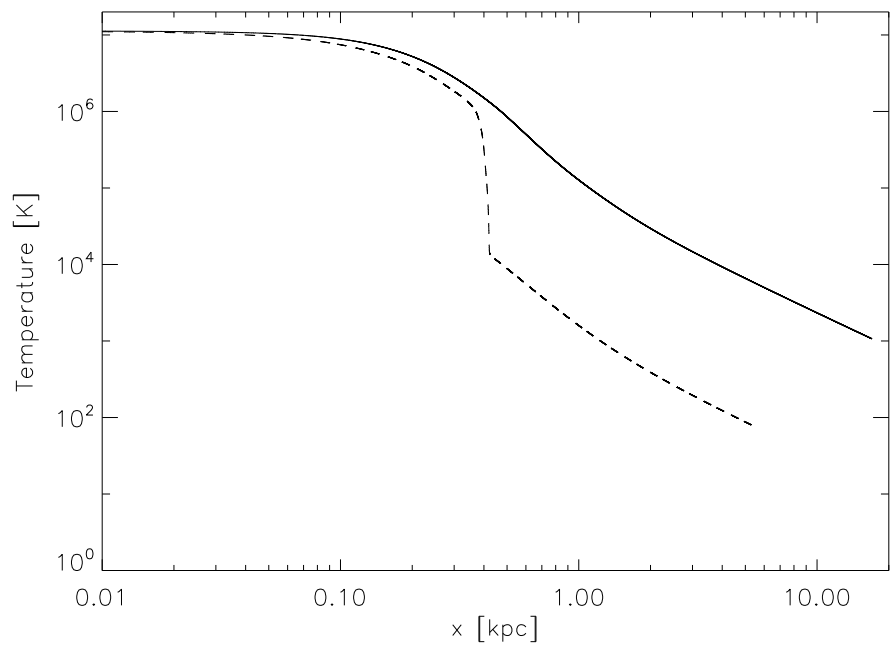

Fig. 5. The gas temperature as a function of height $x$ above the galactic plane. The lines have the same description as Fig. 2.

the gravitational force acting directly on the material within the nozzle flow. From Fig. 2, we see that the solution for $Z=5$ (with stronger radiative cooling) shows a sudden collapse of the nozzle radius at $x \approx 40 \mathrm{kpc}$. This collapse is caused by the strong radiative cooling at temperatures below $10^{6} \mathrm{~K}$, and is qualitatively similar to the results found for cooling nozzle flows by Raga \& Cantó (1989). Figure 3 shows the velocity along the nozzle flow for the two models. The flow velocity first grows with increasing $x$, reaches a maximum, and then collapses to zero at the point where the nozzle radius diverges (see Fig. 2). We show in Fig. 4 the density as a function of position along the nozzle flow. For $Z=1$, a monotonically decresing density vs. $x$ relationship is found. For $Z=5$, a sharp increase in the density is found at the position in which the nozzle radius collapses (see Fig. 2).

Finally, in Fig. 5 we show the temperature structure of the nozzle flow. The $Z=5$ model shows a sudden collapse at $x \approx 40 \mathrm{kpc}$, which is absent in the $Z=1$ model. To explore of parameter space, we have run models with $v_{\mathrm{w}}=500-800 \mathrm{~km} \mathrm{~s}^{-1}$ (in $\Delta v_{\mathrm{w}}=50 \mathrm{~km} \mathrm{~s}^{-1}$ steps) and $Z=1-10$ (in $\Delta Z=1$ steps). From these models, we computed the maximum height $x_{\mathrm{m}}$ reached by the nozzle flow. The values obtained for $x_{\mathrm{m}}$ as a function of $v_{\mathrm{w}}$ and $Z$ are shown in Fig. 6.

From this figure, we see that flows with higher wind velocity reach larger heights $x_{\mathrm{m}}$. Higher values of $x_{\mathrm{m}}$ are also obtained for decreasing metallicities. The radiative nozzle flows inject cool material at $x_{\mathrm{m}}$, which will then fall back onto the galactic plane in the form of dense, neutral clumps. When these clumps have reached a height $x_{0}$, they will have the infall velocities given in Fig. 1.

\section{Discussion: low and high velocity $\mathrm{HI}$ clouds}

For the adiabatic case, we obtain nozzle flow solutions that extend out to infinity for cluster wind velicities of $v_{\mathrm{w}}>v_{\mathrm{esc}}$, where $v_{\text {esc }}$ is the escape velocity from the position of the stellar cluster that feeds the flow (see Sect. 3). For $v_{\mathrm{w}}<v_{\mathrm{esc}}$, the flow stalls at a finite distance $x_{\mathrm{m}}$ from the galaxy. As the nozzle stalls, its cylindrical radius diverges, producing a layer of neutrally buoyant gas at a distance $\sim x_{\mathrm{m}}$ from the wind source. If this gas cools radiatively, it will become negatively buoyant and eventually fall back onto the galactic plane, reaching velocities similar to the free-fall velocity (see Fig. 1).

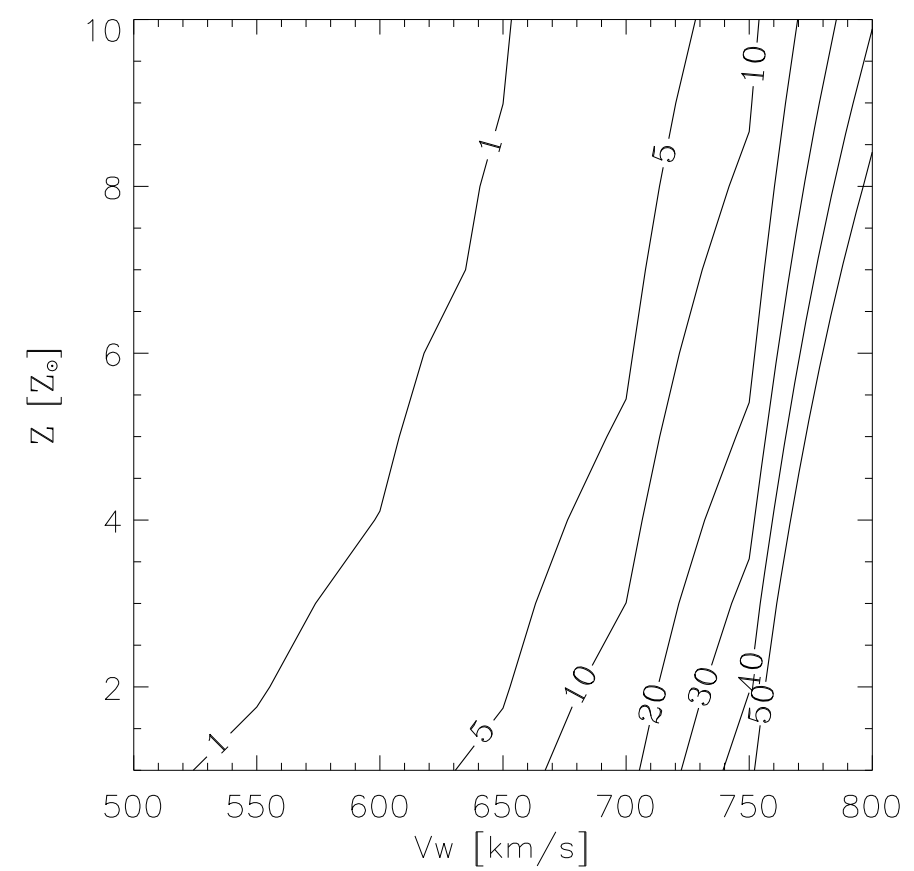

Fig. 6. Isocontours of maximum height $x_{\mathrm{m}}$ (in kpc) reached by the nozzle flow as function of the metallicity $Z$ and cluster wind $V_{\mathrm{w}}$.

We then integrated the equations numerically for a radiative nozzle flow. We included the cooling function of Raymond et al. (1976) tabulated as a function of temperature for a set of different metallicities (assuming a "low density regime" $\propto \rho^{2}$ density dependence). We assume that the nozzle flow propagates away from the central region of our galaxy, within an isothermal halo subject to the Galactic potential of Espresate et al. (2002) and Allen \& Santillán (1991).

We have run a grid of radiative nozzle models for cluster winds with velocities $500 \leq v_{\mathrm{w}} \leq 800 \mathrm{~km} \mathrm{~s}^{-1}$ and metallicities $1<Z<10 Z_{\odot}$. From these models, we found that the radiative cooling lowers the height $x_{\mathrm{m}}$ at which the nozzle flow stalls. Therefore, for higher material metallicities within the nozzle flows (resulting in higher cooling rates), we obtained lower values of $x_{\mathrm{m}}$ (see Fig. 6).

In the models with $Z=1 Z_{\odot}$, as the cluster wind velocity increases from $v_{\mathrm{w}}=500$ to $750 \mathrm{~km} \mathrm{~s}^{-1}$, the value of $x_{\mathrm{m}}$ increases from 1 to $50 \mathrm{kpc}$. For higher values of $v_{\mathrm{w}}$, the value of $x_{\mathrm{m}}$ rapidly diverges, indicating that the nozzle flows do not stall (and therefore never produce negatively buoyant material falling back onto the galaxy).

In the models with $Z=5 Z_{\odot}$, the same range of $x_{\mathrm{m}}$ values (i.e., from 1 to $50 \mathrm{kpc}$ ) is obtained for cluster wind velocities ranging from $v_{\mathrm{w}}=620$ to $770 \mathrm{~km} \mathrm{~s}^{-1}$. For $v_{\mathrm{w}}$ approaching $1000 \mathrm{~km} \mathrm{~s}^{-1}$, the nozzle flows never stall, regardless of the metallicity.

Therefore, from our models we could obtain nozzle flows that stall at heights ranging from 1 to $50 \mathrm{kpc}$ for cluster wind velocities ranging from $\sim 500$ to $800 \mathrm{~km} \mathrm{~s}^{-1}$ (and solar abundances $Z=1-10)$.

Let us now consider low velocity clouds, which are observed at heights $x_{0} \sim 0.3-2.5 \mathrm{kpc}$ and have infall velocities $<100 \mathrm{~km} \mathrm{~s}^{-1}$. From Fig. 1, we see that these clouds could only have originated in initial heights, $x_{\mathrm{m}}<4$. From Fig. 6 . we see that this height corresponds to the stalling distances of nozzles with cluster wind velocities $v_{\mathrm{w}}<620 \mathrm{~km} \mathrm{~s}^{-1}$ for $Z=1 Z_{\odot}$ and $v_{\mathrm{w}}<720 \mathrm{~km} \mathrm{~s}^{-1}$ for $Z=10 Z_{\odot}$. High velocity clouds 
are observed at heights $x_{0}>12 \mathrm{kpc}$ and have infall velocities $100 \rightarrow 200 \mathrm{~km} \mathrm{~s}^{-1}$. From Fig. 1, we see that clouds with infall velocities of $150 \mathrm{~km} \mathrm{~s}^{-1}$ at $x_{0}=15 \mathrm{kpc}$ (these being representative values for high velocity clouds) have originated at initial heights $x_{\mathrm{m}} \approx 26 \mathrm{kpc}$. From Fig. 6, we see that this height corresponds to the stalling heights of nozzles with $v_{\mathrm{w}}=720 \mathrm{~km} \mathrm{~s}^{-1}$ (for $Z=1 Z_{\odot}$ ) up to $v_{\mathrm{w}}=780 \mathrm{~km} \mathrm{~s}^{-1}$ (for $Z=10 Z_{\odot}$ ).

In this way, we see that a sequence of nozzle models of increasing $v_{\mathrm{w}}$ with $500<v_{\mathrm{w}}<800 \mathrm{~km} \mathrm{~s}^{-1}$ naturally explains the range of infall velocities observed from low to high velocity HI clouds. Nozzle flows produced by cluster winds with higher $v_{\mathrm{w}}$ do not stall, and therefore will not produce large amounts of negatively buoyant material at high altitudes that could form the observed HI clouds.

\section{Conclusions}

We have presented a de Laval nozzle model for galactic fountain flows. In this flow, the wind emitted (in our case) by a stellar cluster is thermalized in a wind shock, and is then reaccelerated out of the galactic plane into a collimated flow as a result of the pressure stratifiction of the surrounding environment.

The equations for this kind of flow were first studied in the context of the production of jet flows from AGN by Blandford \& Rees (1974), and in the context of jets from young stars by Königl (1982). Raga \& Cantó (1989) studied the effect of radiative cooling within the nozzle flow (also in the context of jets from young stars).

We have written the equations for a nozzle flow, including both a radiative cooling term and the action of the gravitational force on the material within the nozzle flow. This latter effect is caused because the gravitational force (in our case, because of the Galactic potential) producing the environmental stratification also acts directly on the material flowing along the nozzle. This effect has not been included in any of previously published astrophysical nozzle flow models.

From numerical integrations of the radiative nozzle flow equations, we found that cluster winds with $600<v_{\mathrm{w}}<$ $750 \mathrm{~km} \mathrm{~s}^{-1}$ produce nozzles that stall at heights $3<x_{\mathrm{m}}<50 \mathrm{kpc}$ above the galactic centre (see Fig. 6). The negatively buoyant clumps that become separated from the stalled flows fall back towards the galactic plane at velocities $<200 \mathrm{~km} \mathrm{~s}^{-1}$ (see Fig. 1). This velocity range covers the infall velocities found for both low and high velocity HI clouds.

From this study, we conclude that the high and low velocity clouds observed to be falling onto the Galactic disk could indeed be produced by winds from massive stellar clusters close to the Galactic centre. The condition for these clusters to produce the observed clouds is that the cluster wind velocity $v_{\mathrm{w}}$ should be lower than $\sim 750 \mathrm{~km} \mathrm{~s}^{-1}$, with a somewhat higher or lower velocity limit depending on the metallicity $Z$ of the gas in the cluster winds (see Fig. 6). Clusters such as the Arches (Stevens $\&$ Hartwell 2003) and the Quintuplet clusters (Rockefeller et al. 2005) are clear candidates for driving this type of nozzle flow.

The Arches and Quintuplet clusters eject masses of the order of the mass in an IVC (see Sect. 5). Therefore, several clusters such as these are necessary to produce an appreciable fraction of all observed IVC's. A population of this clusters might be present in the Galactic centre (with several still undetected clusters). On the other hand, a complex of HVC's has a combined mass of $\sim 10^{5}-10^{6} M_{\odot}$ (see Sects. 1,5$)$. To eject these masses from the Galaxy, we would need considerably more massive SSC's (with $\sim 10^{4}-10^{5}$ massive stars). It appears unlikely that if these clusters were present around the Galactic centre they would not have yet been detected. Therefore, it appears more likely that these clusters (if they exist) are located far away from the Galactic centre. It would therefore be interesting to explore models of nozzle flows produced by clusters at some distance from the Galactic centre.

We should mention the work of Wünsch et al. (2008). These authors showed that the presence of thermal instabilites inside the wind from a very massive SSC (with more than $8 \times 10^{6}$ solar masses) results in a substantial decrease in the total mass-loss rate of the cluster wind. This effect would serve to moderate the strength of fountain flows produced by these clusters.

To proceed with this study, it will be necessary to remove at least some of the simplifying assumptions of our present model. For example, we have assumed that the nozzle flow has a uniform velocity across the nozzle cross-section. The removal of this assumption would probably lead to flows in which an outer, slower envelope could stall, while a higher velocity core (moving more repidly than the escape velocity) could continue flowing out to large distances from the Galaxy. In this a model, cluster winds with higher values of $v_{\mathrm{w}}$ might be able to produce nozzles that form negatively buoyant, infalling clumps.

Inn our work, we have also assumed that the cluster producing the nozzle flow is located close to the Galactic centre. It would be interesting to study off-centre cluster winds, which should produce nozzle flows with curved paths, because HVC's could indeed be formed by massive clusters at some distance from the Galactic centre (see above).

Finally, it would also be interesting to study the spatial distribution of the infalling clumps (produced by a nozzle flow) on the galactic plane. To address these issues, it will probably be necessary to carry out full, 3D gasdynamic simulations similar to those of de Gouveia Dal Pino et al. (2009, who considered the production of high and low velocity clouds by SN explosions).

Acknowledgements. We acknowledge support from the CONACyT grant 61547. We thank an anonymous referee for helpful comments. A.C.R. acknowledges Steve Shore for interesting discussions ( 20 years ago) on the subject of buoyancy in nozzle flows.

\section{References}

Allen, C., \& Santillan, A. 1991, RMxAA, 22, 255

Blandford, R. D., \& Rees, M. F. 1974, MNRAS, 169, 395

Blitz, L., Spergel, D. N., Teuben, P. J., Hartmann, D., \& Burton, W. B. 1999, ApJ, 514, 818

Bluhm, H., de Boer, K. S., Marggraf, O., \& Richter, P. 2001, A\&A, 367, 299

Cotera, A. S., Erickson, E. F., Colgan, S. W. J., et al. 1996, ApJ, 461, 750

de Gouveia Dal Pino, E. M., Melioli, C., D’Ercole, A., Brighenti, F., \& Raga, A. 2009, RMAAxC, in press

Dettmar, R. J. 2005, in Magnetic Fields in the Universe: from Laboratory and Stars to Primordial Structures, ed. E.M. de Gouveia Dal Pino, G. Lugones, \& A. Lazarian, AIP Proc., 784, 354

De Young, D., \& Heckman, T. 1994, ApJ, 431, 598

Dickey, J. M., \& Lockman, F. J. 1990, ARA\&A, 28, 215

Espresate, J., Cantó, J., \& Franco, J. 2002, ApJ, 575, 194

Francois, L., Tenorio-Tagle, G., Silich, S., Kunth, D., \& Cerviño, M. 2001, ApJ, 560,630

Fraternali, F., \& Binney, J. J. 2006, MNRAS, 366, 449

Gallager, J., \& Smith, L. 1999, MNRAS, 304, 540

Garmire, G. P., Nousek, J. A., Apparao, K. M. V., et al. 1992, ApJ, 399, 694

Heckman, T. M., Armus, L., \& Miley, G. K. 1990, ApJS, 74, 833

Houck, J. C., \& Bregman, J. N. 1990, ApJ, 352, 506

Königl, A. 1982, ApJ, 261, 115 
Konstantopoulos, I. S., Bastian, N., Smith, L. J., et al. 2008, ApJ, 674, 846 Leitherer, C., \& Heckman, T. M. 1995, ApJS, 96, 9

Lu, L., Savage, B. D., Sembach, K. R., et al. 1998, AJ, 115, 162

Melioli, C., Brighenti, F., D’Ercole, A., \& de Gouveia Dal Pino, E. M. 2008, MNRAS, 388, 573

Melo, V. P., Muñoz-Tuñón, C., Maíz-Apellániz, J., \& Tenorio-Tagle, G. 2005, ApJ, 619, 270

Meynet, G., \& Maeder, A. 2002, A\&A, 390, 561

Münch, G., \& Zirin, H. 1961, ApJ, 133, 11

Murphy, E. M., Sembach, K. R., Gibson, B. K., et al. 2000, ApJ, 538, 35

Nagata, T., Woodward, C. E., Shure, M., \& Kobayashi, N. 1995, AJ, 109, 1976

Oort, J. H. 1970, A\&A, 7, 381

Raga, A. C., \& Cantó, J. 1989, ApJ, 344, 404

Raymond, J. C., Cox, D. P., \& Smith, B. W. 1976, ApJ, 204, 290

Rockefeller, G., Fryer, C. L., Melia, F., \& Wang, Q. D. 2005, ApJ, 623, 171
Sembach, K. R. 2000, ApJ, 538, L31

Serabyn, E., Shupe, D., \& Figer, D. F. 1998, Nature, 394, 448

Shapiro, P. R., \& Fields, G. B. 1976, ApJ, 205, 762

Silich, S., Tenorio-Tagle, G., \& Rodríguez-González, A. 2004, ApJ, 610, 226

Stevens, I. R., \& Hartwell, J. M. 2003, MNRAS, 229, 280

Tenorio-Tagle, G., Silich, S., \& Muñoz-Tuñón, C. 2003, ApJ, 597, 279

Veilleux, S., Cecil, G., \& Bland-Hawthorn, J. 2005, ARA\&A, 43, 769

Wakker, B. P., Savage, B. D., Oosterloo, T. A., \& Putman, M. E. 1999, in The Stromlo Workshop on High-Velocity Clouds, ed. B. K. Gibson, \& M. E. Putman (San Francisco: ASP), ASP Conf. Ser., 166, 302

Wakker, B. P., \& van Woerden, H. 1997, ARA\&A, 35, 217

Walcher, C. J., Böker, T., Charlot, S., et al. 2006, ApJ, 649, 692

Whitmore, B. C., \& Schwizer, F. 1995, AJ, 109, 960

Whitmore, B. C. 2001, in Astrophysical Ages and Times Scales, ASP Conf. Ser., 245,411

Wünsch, R., Tenorio-Tagle, G., Palous, J., \& Silich, S. 2008, ApJ, 683, 692 